\title{
POSITIVE COMMUTATORS AND COLLECTIONS OF OPERATORS
}

\author{
Roman DrnovŠEK, MARTin JESEnKo And Marko KAndić
}

Abstract. Let $A$ and $B$ be completely decomposable nonnegative matrices such that the commutator $A B-B A$ is also a nonnegative matrix. We prove that the set $\{A, B\}$ is completely decomposable, i.e., there exists a permutation matrix $P$ such that $P A P^{-1}$ and $P B P^{-1}$ are upper triangular matrices. We show similar results for collections of completely decomposable nonnegative matrices. We also find conditions on commutators under which a given operator on a Riesz space is necessarily scalar.

Mathematics subject classification (2010): 15A48, 20M20.

Keywords and phrases: Nonnegative matrices, semigroups of matrices, indecomposability, commutators.

\section{REFERENCES}

[1] C. D. Aliprantis, O. Burkinshaw, Positive operators, Springer, 2006.

[2] J. Bračič, R. Drnovšek, Y. B. Farforovskaya, E. L. Rabkin, J. Zemánek, On positive commutators, Positivity 14, 3 (2010), 431-439, DOI: 10.1007/s11117-009-0028-1.

[3] R. DRnOvŠEK, M. KANDIĆ, Ideal-triangularizability of semigroups of positive operators, Integral Equations Operator Theory 64, 4 (2009), 539-552.

[4] H. Radjavi, P. Rosenthal, Simultaneous Triangularization, Springer-Verlag, New York, 2000. 